\title{
Estilos de aprendizagem e solução de problemas: um estudo com pré-escolares
}

\author{
Acácia Aparecida Angeli dos Santos \\ Rogéria Gasparim Amadi \\ Katya Luciane de Oliveira \\ Universidade São Francisco, Atibaia-SP
}

\begin{abstract}
RESUMO
O objetivo deste estudo foi o de averiguar a relação entre o estilo de aprendizagem e a solução de problemas, verificando qual situação de estímulo musical poderia propiciar melhor desempenho na tarefa. Participaram deste estudo 22 crianças entre cinco e seis anos de idade, que responderam oralmente a um inventário de estilo de aprendizagem e resolveram três quebra-cabeças, sob diferentes condições de apresentação do estímulo musical. Os resultados evidenciaram que as crianças alcançaram uma pontuação acima da média em todas as dimensões de estilo avaliadas. A situação de solução de problemas com música lenta foi a que favoreceu a ocorrência do maior número de respostas corretas, sendo significativamente maior que nas demais situações (música agitada e sem música). Observou-se que o estímulo musical, quando adequadamente utilizado, pode ser um elemento facilitador no enfrentamento de situações-problema, independentemente do estilo de aprendizagem da criança.
\end{abstract}

Palavras-chave: estratégias de aprendizagem; música; estilos cognitivos.

\begin{abstract}
Learning styles and problems solving: a study with pre-school children

The objective of this study has been to examine the relation between learning styles and problem solving, verifying which situation of musical stimulus could promote a better performance on preschool children at a given task. A sample of 22 children between five and six years old participated in this study, replying aloud to a learning style inventory read to them by the experimenters, and solved three puzzles under different conditions of musical stimulus. The results certified that participants had a score above average in all learning style dimensions evaluated. The problem-solving situation accompanied by soft music was the one that favored a highest frequency of correct answers, being significantly higher than the remaining situations (e.g., exciting music or no music at all). It has been observed that, when adequately used, musical stimulus could be a facilitating element in facing problem-solving situations, independently of the child's learning style.
\end{abstract}

Keywords: learning strategies; music; cognitive styles.

\section{INTRODUÇÃO}

A aprendizagem pode ser entendida como um processo individual que está permanentemente em construção, levando à integração progressiva do conhecimento. Nessa perspectiva, vários teóricos têm estudado os processos que envolvem as práticas de ensino e aprendizagem, buscando alternativas que facilitem esse processo (Bariani, Sisto \& Santos, 2000; Pitta, Santos, Escher \& Bariani, 2000; Santos, Bariani \& Cerqueira, 2000, entre outros). Psicólogos e educadores estão procurando compreender não apenas como o indivíduo utiliza sua inteligência, mas também os mecanismos de processamento de informação envolvidos (Black, 2004; Cerqueira, 2000; Honigsfeld \& Dunn, 2003; Jongsma, 1990; Rosa \& Santos, 2001).
Considerando a educação sob esse aspecto, devem ser oferecidos à escola todos os recursos possíveis para conhecer mais sobre o processo de aprendizagem, incluindo a forma com que o aprendiz lida com situações novas. Dessa forma, o professor estará mais capacitado para ser um facilitador mais eficaz em suas práticas educativas. Cada vez mais se fortalece a idéia de que cada estudante possui uma maneira particular e individual de adquirir conhecimento e processar informações, o que é denominado de estilo de aprendizagem ou mesmo de estilos cognitivos (Messick, 1994; Schmeck \& Geisler-Brenstein,1989, entre outros).

O surgimento das concepções sobre esse construto, isto é, da necessidade de compreensão da interação do indivíduo com o ambiente de aprendizagem em que está inserido, estabeleceu-se a partir das pesquisas 
realizadas pelos estudiosos do processamento humano da informação. Enfatizando principalmente o como se aprende, essas pesquisas trouxeram novas perspectivas para a compreensão do processo de aprendizagem e de solução de problemas (Cano-Garcia \& Hughes, 2000; Cerqueira \& Santos, 2000, Riding, Grimley, Dahraei \& Banner, 2003).

Entretanto, quando se aborda o conceito estilo de aprendizagem, freqüentemente surge também referência ao estilo cognitivo. Os estudos a respeito desses estilos foram realizados a partir do interesse em conhecer as diferenças individuais, como o de Galton em 1883 e o de Allport em 1937, conforme observado por Santos e cols (2000). No entanto, é difícil uma definição exata e uma operacionalização desses conceitos, uma vez que derivam de diversos referenciais teóricos, advindos das abordagens cognitiva, comportamental, gestáltica e da psicanálise, entre outras.

Para Riding e Cheema (1991) o construto estilo de aprendizagem surgiu na década de 70 como um termo substituto do termo estilo cognitivo, aparentemente mais relacionado a questões teóricas e acadêmicas. Para eles, o termo estilo de aprendizagem apareceu sempre mais associado a aplicações práticas. Todavia, tal diferenciação é muito frágil, visto que as pesquisas sobre estilos de aprendizagem apresentam fundamentos nas teorias de estilos cognitivos, o que pressupõe que também possuem questões teóricas subjacentes. Também foi constatado que não apenas os estilos de aprendizagem possuem aplicações práticas, mas os estilos cognitivos também.

Os termos estilo cognitivo e de aprendizagem estão sendo utilizados por pesquisadores e teóricos sem uma discriminação entre eles, ocorrendo muitas conceitualizações para cada um deles, conforme observa Santos e cols. (2000). Os estilos de pensar e aprender se propõem a explicar a forma como se percebe, processa e se transforma a informação. Uma das diferenciações mais aceita entre eles é a de que os estilos cognitivos correspondem a estratégias de processamento de informações para a solução de problemas, voltados para o domínio da percepção visual. Já, os estilos de aprendizagem correspondem à maneira que as pessoas interagem com as condições de aprendizagem, compreendendo os aspectos cognitivos, afetivos, físicos e ambientais, responsáveis por favorecer o processamento de informações.

O desenvolvimento desses estilos ocorre por meio da utilização repetida de determinadas estratégias, na execução de tarefas diferentes, com exigências e condições adaptativas semelhantes. Tanto os estilos cognitivos, quanto os estilos de aprendizagem abrangem capacidades de atenção, motricidade, percepção, pensamento, memória, aprendizagem e solução de problemas. Constituem padrões de adaptação típicos, próprios dos seres humanos, inferidos por meio de observação. Apesar disso, Santos e cols. (2000) salientam que existem poucos estudos que relacionam os resultados quantitativos e qualitativos da aprendizagem com os dois conceitos simultaneamente, sendo difícil avaliar o que se deve a um ou ao outro.

Rosa e Santos (2001) entendem que a educação influencia na formação da atitude e da orientação do aluno por meio da aprendizagem, uma vez que estabelece atitudes positivas na solução de problemas. Nesse sentido, a experiência educacional, desde seu início, ajuda a formar o estilo de aprendizagem pessoal. Desse modo, é possível afirmar que o estilo de aprendizagem é desenvolvido a partir das vivências do seu dia-a-dia, sendo que ao interagir com o mundo, o indivíduo pouco a pouco constrói sua maneira peculiar de experienciá-lo e de lidar com novas situações.

\section{Estilos de aprendizagem, solução de problemas e música}

Entende-se por problemas, situações específicas de vida que exigem respostas adaptativas, mas que não recebem respostas eficazes de enfrentamento devido a alguns obstáculos. Os problemas são uma discrepância entre a realidade de uma situação e os objetivos (Nezu \& Nezu, 1999). Conseqüentemente, levam a um desequilíbrio na relação entre a pessoa e o ambiente que a cerca, fazendo com que o indivíduo não possua as respostas adaptativas desejadas.

A busca pela solução desses problemas diz respeito à tentativa de uma resposta de enfrentamento que modifique a situação problema, ocasionando uma modificação de comportamento. Dessa forma, uma solução, dita eficaz, consiste numa resposta que atinja esses objetivos, maximizando as consequiências positivas e, ao mesmo tempo, minimizando as negativas (Caballo, 1999).

A solução de problemas pertence ao campo cognitivo do ser humano que, no caso da criança, tem seu desenvolvimento ampliado por meio da atividade lúdica. De acordo com Salvucci (1997), o brincar influencia o desenvolvimento da criança, visto que a motiva a trabalhar em sala de aula e fornece as habilidades necessárias ao desenvolvimento do raciocínio, aprimorando a capacidade crítica e a sua formação intelectual. Ressalta ainda, que na sala de aula, por meio do brincar, a criança articula seu mundo imaginário e o mundo real. Nesse sentido, a música pode ser considerada como uma atividade lúdica benéfica para o desenvolvimento infantil. Assim sendo, hipotetiza-se que o recurso musical possa auxiliar na solução de problemas e contribuir para o processo de aprendizagem infantil.

Quando se fala em música, deve-se levar em consideração o seu caráter subjetivo, o que torna sua con- 
cepção muito variada ao longo dos tempos. Para Montanari (1993), até alguns anos atrás, a conceituação de música estava associada a uma combinação ordenada e racional de sons. Esses sons musicais podem ser definidos como uma manifestação vibratória, que apresenta definição de freqüência, passível de ser identificada pelo ouvido humano. No entanto, quando essas vibrações são mal definidas, são denominadas como ruídos. Atualmente a música pode ser constituída tanto por sons quanto por ruídos.

Existem dois aspectos do significado musical. O primeiro está associado diretamente à música, como padrões rítmicos, instrumentos utilizados ou estilo; o segundo refere-se aos contextos sociais que influenciam a compreensão musical. A música pode ser utilizada para fornecer alguma informação sobre a classe social, a etnia, religião, valores políticos, entre outras. Dessa forma, é carregada por significados e está relacionada com o aspecto sociocultural (Beineke, 2001).

Figueiredo (1996) considera que a linguagem musical pode e deve ser aprendida concomitantemente com a linguagem falada, favorecendo o desenvolvimento infantil. Ressalta que ela estimula o desenvolvimento da receptividade oral, da memória, das respostas físicas e emocionais, da percepção auditiva, da imaginação, de habilidades que envolvem seqüenciação e a linguagem corporal, dentre outros. Considerando os aspectos psicológicos, o autor entende que a música possui uma conotação emocional para cada indivíduo, sendo possível se expressar por meio dela.

Empregando a ópera na iniciação à leitura e à escrita, Pellegrini (2000) mostrou que a utilização da música pode ser um elemento facilitador para um bom desempenho. Corroborando essa informação, outros pesquisadores como Silva (1998) e Corrêa (2002) também utilizaram a música na aprendizagem em projetos de diferentes naturezas, como por exemplo, no ensino da língua portuguesa.

Investigando os efeitos da música de fundo sobre o comportamento de crianças pré-escolares durante o recreio e em sala de aula Marquetti (1994) constatou que a estimulação musical aumentou a ocorrência de movimentos rítmicos (ritmicidade referente à movimentação individual da criança). Verificou, também, a ocorrência de padrões interativos não-verbais (movimentação socialmente dirigida da criança) e de outros eventos interativos (olhar prévio e contato da criança com seus pares) tanto na situação de recreio, quanto na sala de aula. Ao lado disso, os padrões verbais dos alunos (movimentação dirigida socialmente) aumentaram na sala de aula. No mesmo sentido, Figueiredo (1996) observou que a música parece proporcionar às crianças um ambiente favorável para a aprendizagem, uma vez que as motiva para a ação.

Considerando o exposto, tanto em relação aos estilos de aprendizagem como em relação à música, a presente pesquisa buscou averiguar se o recurso musical pode ser usado como elemento facilitador no enfrentamento de situações novas. Para tanto, foi realizada a identificação dos estilos de aprendizagem das crianças submetidas a atividades que envolvem a solução de problemas e a comparação do desempenho das crianças em diferentes condições de estímulo ambiental na execução dessas tarefas.

\section{MÉTODO}

\section{Participantes}

Participaram 22 alunos pertencentes a uma escola municipal de educação infantil, do interior do estado de São Paulo, cujo nível socioeconômico era baixo. A média de idade foi de 5 anos e cinco meses $(D P=0,5)$. $\mathrm{O}$ gênero masculino representou $45,5 \%(N=10)$ das crianças e o feminino $54,5 \%(N=12)$.

As crianças foram dividas aleatoriamente em três grupos, por meio de sorteio. Dois deles foram constituídos por oito crianças e um por seis crianças. Para a execução da tarefa também houve uma divisão aleatória de duplas. Desse modo, o Grupo 1 foi composto por $36,4 \%(N=8)$ das crianças, o Grupo 2 por $27,3 \%$ $(N=6)$ e o Grupo 3 por $36,4 \%(N=8)$.

\section{Material}

(a) Seis quebra-cabeças de madeira, constituídos de 12 peças e apropriados à idade das crianças em questão, sendo que foram montados dois quebracabeças em cada situação, em três momentos diferentes. Os utilizados na Situação A envolviam a figura de uma borboleta (Q.1) e de uma tartaruga (Q.2); os da Situação B correspondiam a desenhos de um caminhão (Q.3) e de baleias (Q.4); os da Situação C à forma de um cachorro (Q.5) e de patos (Q.6).

(b) Um cronômetro da marca Sunway S-1045. O instrumento foi utilizado para medir o tempo de realização da tarefa, preestabelecido para 5 minutos.

(c) Duas músicas distintas, selecionadas previamente: uma com ritmo lento e outra com ritmo acelerado (agitado), representadas pela Sonata $n^{o} 8$ in $C$ Minor, Op. 13 'Pathétique' (Adágio cantabile) e a Razumovsky String Quartet Op. 59, $n^{\circ} 2$ (Presto più presto), ambas do compositor Beethoven (CD gravado em 1998).

(d) O inventário de estilos de aprendizagem infantil, adaptado por Amadi e Santos (2003), baseados nos estilos de pensar e aprender de Squarizi (1999), com o propósito de identificar o estilo preferencial de pensar e aprender de crianças entre 5 a 10 anos de idade, resultou em um instrumento constituído por 53 ques- 
tões fechadas, que abordaram as dimensões ambientais, emocionais e sociológicas, apresenadas a seguir.

A dimensão ambiental é constituída pelas seguintes áreas: som, luz, temperatura, ambiente e parte do dia (15 questões). Um exemplo típico de item que avalia essa dimensão pode ser ilustrado pelo item 5 "Consigo me concentrar em ambientes barulhentos sem problema".

Já a dimensão emocional é composta pelas áreas motivação, persistência, responsabilidade e estrutura (15 questões). Como exemplo de item, pode-se citar o de número 27 "Eu me interesso pelas coisas que aprendo na escola".

Finalmente a dimensão sociológica é constituída pelas áreas individual ou grupal, com professor/adulto, visão, audição, gustação, tato, cinestesia e mobilidade (23 questões). Para exemplificar, pode-se citar o item 37 "Não gosto de fazer trabalhos em grupo".

\section{Critérios de Avaliação}

Os pontos obtidos no inventário de estilos de aprendizagem foram aferidos com base na freqüência de respostas das crianças, sendo que poderiam responder as alternativas, sempre, às vezes ou nunca. Foi atribuída a essas respostas a seguinte pontuação: 2,1 e 0 , respectivamente. Quatro itens tiveram sua pontuação invertida na dimensão ambiental; oito na dimensão emocional e 11 na dimensão sociológica, para que mantivessem o sentido esperado das respostas, ou seja, quanto maior a pontuação maior a predominância de uma dimensão no estilo de aprendizagem do sujeito.

Com relação à situação-problema, ou seja, a solução dos quebra-cabeças, foi considerada como correta somente as peças que estavam exatamente encaixadas, sendo atribuído um ponto para cada acerto. É importante ressaltar que não foram pontuadas as tentativas de encaixe que ficaram apenas parcialmente encostadas, mesmo que as peças escolhidas fossem as adequadas.

\section{Procedimento}

Inicialmente os responsáveis pelas crianças assinaram um termo de consentimento esclarecido, documentando que eles permitiram a execução da pesquisa. Foi realizada a montagem individual de três quebra-cabeças, sendo que foram montados em dias e em três situações diferentes. Em cada uma das situações, os quebra-cabeças possuíam o mesmo nível de dificuldade e durante a realização da atividade, as crianças foram subdividas em duplas, mas executaram a tarefa individualmente, com um tempo de duração pré-fixado de cinco minutos.

$\mathrm{Na}$ Situação A foi realizada a montagem dos quebra-cabeças Q.1 e Q.2, ouvindo uma música agitada. $\mathrm{Na}$ Situação B ocorreu a montagem dos quebracabeças Q.3 e Q.4, ouvindo uma música lenta, e na Situação C houve a montagem dos quebra-cabeças Q.5 e Q.6, sem a apresentação de música.

Para controlar o efeito da situação, as duplas foram divididas em três grupos que resolveram os quebracabeças em diferentes sequiências de situação. O Grupo 1 foi submetido à seqüência das situações $A, B$ e C. O Grupo 2 se submeteu à seqüência das situações, $\mathrm{C}, \mathrm{B}$ e $\mathrm{A}$ e o Grupo 3 se submeteu à seqüência das situações $\mathrm{C}$, A e B. Vale salientar que nas três situações, todos os grupos tiveram cinco minutos para montar os quebra-cabeças, sendo cada uma em dias diferentes e consecutivos. As crianças executaram individualmente a tarefa determinada. Posteriormente, a coleta de dados referente ao inventário de estilos de aprendizagem foi realizada por uma das pesquisadoras, de forma individual.

\section{RESULTADOS}

Aplicando-se as provas de estatística descritiva foram obtidos as médias, desvios padrão e pontuação mínima e máxima em relação aos estilos de aprendizagem, em cada dimensão e no total. Os resultados encontram-se na Tabela 1.

Tabela 1: Estatísticas descritivas dos estilos de aprendizagem $(N=22)$

\begin{tabular}{lccccc}
\hline Dimensões & Média & $\begin{array}{c}\text { Desvio } \\
\text { Padrão }\end{array}$ & Mínimo & Máximo & $\begin{array}{c}\text { Média } \\
\text { Ponderada }\end{array}$ \\
\hline Ambiental & 17,05 & 3,02 & 10 & 23 & 1,14 \\
Emocional & 19,45 & 3,49 & 13 & 28 & 1,30 \\
Sociológica & 27,27 & 3,47 & 22 & 35 & 1,19 \\
Total & 63,77 & 5,64 & 54 & 74 & 1,20 \\
\hline
\end{tabular}

Também, no que tange à solução do problema de juntar as peças do quebra-cabeça, foram efetuadas provas de estatística descritiva para análise dos dados.
Os acertos obtidos nas diferentes situações podem ser observados na Tabela 2. 
Tabela 2: Estatística descritiva do desempenho na resolução de problemas em cada situação $(N=22)$

\begin{tabular}{|c|c|c|c|c|}
\hline Situação & $\begin{array}{c}\text { Número } \\
\text { mínimo } \\
\text { de acertos }\end{array}$ & $\begin{array}{c}\text { Número } \\
\text { máximo } \\
\text { de acertos }\end{array}$ & $\begin{array}{c}\text { Média } \\
\text { de } \\
\text { acertos }\end{array}$ & $D P$ \\
\hline A - música agitada & 0 & 10 & 2,59 & 3,26 \\
\hline B - música lenta & 0 & 12 & 3,68 & 3,16 \\
\hline $\mathrm{C}$ - sem música & 0 & 9 & 3,18 & 2,78 \\
\hline
\end{tabular}

Vale destacar que foi na Situação A (música agitada) que as crianças apresentaram a menor média de acertos. Pelo teste não paramétrico Kendall's $W$ verificou-se que as diferenças entre os resultados nas situações eram estatisticamente significativas $\left[X^{2}(2,20)=\right.$ $7,618 ; p=0,022]$.
Para constatar se havia diferença no desempenho nas Situações A, B e C, considerando a ordem em que as crianças foram submetidas à situação problema, recorreu-se a Prova $Q$ de Cochran. Observou-se que a maior incidência de acertos foi na Situação B (música lenta), independentemente da ordem em que os grupos foram submetidos. Os resultados foram elencados na Tabela 3.

Tabela 3: Distribuição da comparação entre grupos, em razão da ordem em que foram submetidos à situação problema $(N=22)$

\begin{tabular}{lccc}
\hline \multicolumn{1}{c}{ Situação } & Grupo & $f$ & $\begin{array}{c}\text { Rank de } \\
\text { Médias }\end{array}$ \\
\hline A - Música agitada & 1 & 8 & 11,63 \\
& 2 & 6 & 9,50 \\
& 3 & 8 & 12,88 \\
\hline B - Música lenta & 1 & 8 & 11,63 \\
& 2 & 6 & 11,33 \\
& 3 & 8 & 11,50 \\
\hline C- Sem música & 1 & 8 & 11,69 \\
& 2 & 6 & 10,67 \\
& 3 & 8 & 11,94 \\
\hline
\end{tabular}

Para verificar se havia correlação entre o desempenho na solução de problemas nos três tipos de situa- ções, recorreu-se à prova de Spearman. Os dados resultantes dessa análise estão apresentados na Tabela 4.

Tabela 4: Índices de correlação entre os desempenhos obtidos nas situações musicais $(N=22)$

\begin{tabular}{lccc}
\hline \multicolumn{1}{c}{ Correlações } & $\begin{array}{c}\text { Situação A } \\
\text { música agitada }\end{array}$ & $\begin{array}{c}\text { Situação } B \\
\text { música lenta }\end{array}$ \\
\hline Situação B & $r$ & 0,43 & \\
música lenta & $p$ & 0,04 & \\
Situação C & $r$ & 0,55 & 0,66 \\
sem música & $p$ & 0,01 & 0,00 \\
\hline
\end{tabular}

Os dados evidenciaram que o desempenho dos sujeitos na Situação A foi o que apresentou menor correlação com o desempenho nas outras situações. Verificou-se ainda que a maior correlação obtida foi entre os resultados das Situações B e C.

Pelas médias ponderadas, que implicou a divisão dos pontos pelo número de itens, foi possível verificar-se que a maior susceptibilidade das crianças ocorreu na dimensão emocional, cuja média foi a mais elevada, quando comparada às demais dimensões. Recorrendo-se à prova não paramétrica de Kendall's W para amostras relacionadas, verificou-se que as diferenças detectadas não eram estatisticamente significativas $\left[X^{2}(3,19)=7,0,009 ; p=0,072\right]$.

Considerando que as diferentes condições de sonoridade nas situações-problema enfrentadas pelos participantes, decidiu-se avaliar as crianças em razão do desempenho apresentado na solução dos quebracabeças. Assim, foram separados os participantes com pontuações diferentes (com até um ponto e com quatro pontos ou mais) e comparados em razão dos escores obtidos nas dimensões do inventário de estilos de aprendizagem. Utilizou-se a prova não paramétrica $U$ de Mann-Whitney para amostras independentes, cujos resultados estão apresentados na Tabela 5. 
Tabela 5: Comparação dos grupos extremos por desempenho nas situações problemas e suas pontuações nas dimensões no inventário de estilos de aprendizagem

\begin{tabular}{|c|c|c|c|c|c|c|}
\hline Dimensões & Situação A & $\mathbf{N}$ & $\begin{array}{c}\text { Média } \\
\text { por Rank }\end{array}$ & $\begin{array}{c}\text { Soma } \\
\text { dos Ranks }\end{array}$ & $Z$ & $p$ \\
\hline \multirow[t]{2}{*}{ Ambiental } & até 1 ponto & 14 & 11,93 & 167,00 & $-2,53$ & 0,01 \\
\hline & 4 pontos ou mais & 5 & 4,60 & 23,00 & & \\
\hline \multirow[t]{2}{*}{ Emocional } & até 1 ponto & 14 & 10,79 & 151,00 & $-1,03$ & 0,30 \\
\hline & 4 pontos ou mais & 5 & 7,80 & 39,00 & & \\
\hline \multirow[t]{2}{*}{ Sociológica } & até 1 ponto & 14 & 10,89 & 152,50 & $-1,16$ & 0,25 \\
\hline & 4 pontos ou mais & 5 & 7,50 & 37,50 & & \\
\hline \multirow[t]{3}{*}{ Total } & até 1 ponto & 14 & 11,96 & 167,50 & $-2,56$ & 0,01 \\
\hline & 4 pontos ou mais & 5 & 4,50 & 22,50 & & \\
\hline & Situação B & & & & & \\
\hline \multirow[t]{2}{*}{ Ambiental } & até 1 ponto & 3 & 9,17 & 27,50 & $-1,49$ & 0,14 \\
\hline & 4 pontos ou mais & 9 & 5,61 & 50,50 & & \\
\hline \multirow[t]{2}{*}{ Emocional } & até 1 ponto & 3 & 3,83 & 11,50 & $-1,49$ & 0,14 \\
\hline & 4 pontos ou mais & 9 & 7,39 & 66,50 & & \\
\hline \multirow[t]{2}{*}{ Sociológica } & até 1 ponto & 3 & 7,50 & 22,50 & $-0,56$ & 0,58 \\
\hline & 4 pontos ou mais & 9 & 6,17 & 55,50 & & \\
\hline \multirow[t]{4}{*}{ Total } & até 1 ponto & 3 & 6,67 & 20,00 & $-0,09$ & 0,93 \\
\hline & 4 pontos ou mais & 9 & 6,44 & 58,00 & & \\
\hline & & & & & \multicolumn{2}{|c|}{ cont. } \\
\hline & Situação $C$ & & & & & \\
\hline \multirow[t]{2}{*}{ Ambiental } & até 1 ponto & 8 & 8,44 & 67,50 & $-0,41$ & 0,68 \\
\hline & 4 pontos ou mais & 7 & 7,50 & 52,50 & & \\
\hline \multirow[t]{2}{*}{ Emocional } & até 1 ponto & 8 & 6,75 & 54,00 & $-1,17$ & 0,24 \\
\hline & 4 pontos ou mais & 7 & 9,43 & 66,00 & & \\
\hline \multirow[t]{2}{*}{ Sociológica } & até 1 ponto & 8 & 10,19 & 81,50 & $-2,04$ & 0,04 \\
\hline & 4 pontos ou mais & 7 & 5,50 & 38,50 & & \\
\hline \multirow[t]{2}{*}{ Total } & até 1 ponto & 8 & 8,50 & 68,00 & $-0,47$ & 0,64 \\
\hline & 4 pontos ou mais & 7 & 7,43 & 52,00 & & \\
\hline
\end{tabular}

Os resultados indicam que na Situação A as crianças, separadas pela pontuação obtida na solução dos quebra-cabeças, apresentaram diferenças significativas na pontuação da dimensão ambiental e no escore total. Na Situação B, não se observou diferença em nenhuma das dimensões e na Situação $\mathrm{C}$ a diferença apareceu na dimensão sociológica.

\section{DISCUSSÃO}

Partiu-se do pressuposto de que os estilos de aprendizagem referem-se à maneira pela qual as pessoas interagem com as condições de aprendizagem, envolvendo aspectos cognitivos, afetivos, físicos e ambientais. Dessa forma, o presente estudo foi realizado para avaliar se alterações em um desses aspectos (especificamente o ambiental) poderiam interferir na solução de uma dada situação problema (a montagem de um quebra-cabeças).

Com relação à identificação dos estilos de aprendizagem e considerando-se o escore máximo possível em cada dimensão, verificou-se que as crianças, aqui estudadas, alcançaram sempre uma pontuação acima da média em todas as dimensões. Embora os participantes fossem provenientes de escolas municipais e de uma classe social desfavorecida, esse fator não foi impeditivo para seu desempenho na escala, visto que o estilo de aprendizagem do indivíduo está relacionado às experiências vivenciadas em seu dia-a-dia, que resultam na construção de sua maneira de aprender e solucionar problemas (Messick, 1994; Rosa \& Santos, 2001; Schmeck \& Geisler-Brenstein, 1989).

A maior pontuação obtida no inventário de estilos de aprendizagem com os participantes do estudo aqui apresentado foi na dimensão emocional. Sob esse 
aspecto Jongsma (1990), Honigsfeld e Dunn (2003) e Black (2004) professam que o estilo de aprendizagem refere-se a um processo de construção pessoal que o estudante utiliza para compreender uma nova informação. Os itens da dimensão emocional sugerem aspectos relacionados à motivação, persistência, responsabilidade e estratégias de enfrentamento. Destaca-se a importância de ser dada maior ênfase ao aspecto emocional, principalmente nos aspectos relacionados à persistência diante de tarefas e à responsabilidade ao aprender. Certamente, o aprimoramento dessa dimensão poderia auxiliar numa aprendizagem mais positiva, sendo um aspecto a ser explorado em estudos futuros, envolvendo participantes de diversas faixas etárias.

Caballo (1999) observa que uma solução eficaz na aprendizagem consiste em uma resposta que atinja os objetivos do aprender. Portanto, a música poderia ser um fator que maximizaria de forma positiva a motivação e o interesse nas diversas atividades relacionadas à solução de problemas, bem como à aprendizagem de forma geral.

Nos resultados obtidos na presente pesquisa, constatou-se que a música agitada foi a que proporcionou a menor média de acertos. Foi observado que as crianças, quando expostas a uma sonoridade suave (lenta), apresentaram um desempenho mais eficaz na solução do problema (montagem do quebra-cabeça). Destacase ainda que a maior correlação obtida foi entre os resultados das Situações B e C. À medida que a situação envolveu a apresentação de música lenta ou a ausência de música, o número de junções corretas aumentou.

Nesse sentido, outros estudiosos consideram que a música atinge diretamente o aspecto emocional do indivíduo, facilitando o raciocínio e seqüenciação das operações (Figueiredo, 1996; Beineke, 2001). Os dados obtidos na pesquisa ora relatada são bastante congruentes com as considerações desses autores, visto que por um lado, os participantes tiveram uma maior pontuação na escala na dimensão emocional; por outro, também obtiveram um melhor desempenho quando a montagem não implicava na audição de uma música agitada. Sugere-se, assim, com base nos dados aqui obtidos, que a música agitada foi um fator dificultador para a solução de problemas, possivelmente por não permitir uma concentração adequada.

Vale lembrar que Corrêa (2002) considera que a música incentiva à reflexão dos fatos, por facilitar a fluência do raciocínio e idéias. Talvez o desempenho das crianças tenha sido melhor com a música lenta por promover um estímulo sonoro agradável, facilitando o raciocínio para a montagem do quebra-cabeça. Marquetti (1994) ressalta que a estimulação musical proporcionada por uma música de fundo aumenta a ocorrência de diversos comportamentos motores, bem como melhora aspectos relacionados à interação social e cognição da criança.

\section{CONSIDERAÇÕES FINAIS}

Santos e cols. (2000) afirmam que a aprendizagem é algo construído e que as estratégias de aprender estão relacionadas ao estilo pessoal de cada um. Para que o professor seja capaz de auxiliar seus alunos, seria recomendável que nos cursos de formação de professores houvesse, no mínimo, uma introdução ao estudo da conceituação de estilos de aprendizagem, buscando prepará-los para conhecerem melhor seus alunos, bem como a si mesmos.

Deve-se ressaltar que meios educacionais demandam determinados tipos de estilos, assim como certas habilidades intelectuais. Apesar disso, nem sempre os estilos exigidos estão de acordo com os estilos dos estudantes, o que pode levar à facilitação ou debilitação do processo de ensino-aprendizagem. Nesse sentido, as instituições escolares deveriam se preocupar mais com os métodos de ensino utilizados e oferecer orientações complementares tanto aos professores quanto aos estudantes, melhorando, assim, as condições oferecidas. Nessa direção, Schmeck e GeislerBrenstein (1989) e Messick (1994) defendem que somente com recursos adequados na escola os professores podem proporcionar aos seus alunos formas mais eficazes de pensar e de aprender.

Nesse contexto, a música poderia ser o agente viabilizador da aprendizagem, principalmente se for utilizado um repertório musical suave. Para Beineke (2001) a música possui vários significados e representações no cotidiano das pessoas e se utilizada de forma adequada pode ser um agente facilitador em diversos contextos que envolvam o raciocínio e a aprendizagem.

Portanto, a expressão musical, assim como o estilo de aprendizagem, são construídos social e culturalmente, estando assim, inseridos no processo de aprendizagem das pessoas. É necessário conceber a música e o estilo de aprender, considerando os significados e valores atribuídos por aqueles que participam desse processo.

\section{REFERÊNCIAS}

Amadi, R. G. \& Santos, A. A. A. (2003). Estímulos musicais e solução de problemas com crianças na educação infantil. Manuscrito não publicado, Universidade São Francisco.

Bariani, I. C. D., Sisto, F. F. \& Santos, A. A. A. (2000). Construção de um instrumento de avaliação de estilos cognitivos. Em F. F. Sisto, E. T. B. Sbarderlini \& R. Primi (Orgs.), Contextos e questões da avaliação psicológica (pp. 173-178). São Paulo: Casa do Psicólogo. 
Beethoven, L. V. (1998). Razumovski String Quartet Op. 59, nº 1 (Adágio molto e mesto). Em L. V. Beethoven Concerto Romântico: Quartetos $n^{\circ} 1$ e $n^{\circ} 2$ e Razumovsky Op. 59. Altaya, 1 CD (ca. 74'), faixa 3 (11' e 5"). Compact disc digital audio.

Beethoven, L. V. (1998). Sonata $\mathrm{n}^{\circ} 14$ in C Sharp Minor (Quase uma fantasia) Op. 27, no 2 (Moonlight) (Presto aditato). Em L. V. Beethoven. Concerto Romântico: Sonatas para piano. Patética, Ao luar, Apassionata. Altaya, 1 CD (ca. 55' e 34', faixa 6 (7' e 16'). Compact disc digital áudio.

Beineke, V. (2001). Funções e significados das práticas musicais na escola. Presença Pedagógica Dimensão, 7(40), 57-65.

Black, J. M. (2004). Assessing learning preferences. Plastic Surgical Nursing, 24(2), 68-69.

Caballo, V. E. (1999). Manual de técnicas de terapia e modificação do comportamento. São Paulo: Santos.

Cano-Garcia, F. \& Hughes, E. H. (2000). Learning and thinking styles: An analysis of their interrelationship and influence on academic achievement. Educational Psychology, 20(4), 413430.

Cerqueira, T. C. S. (2000). Estilos de aprendizagem em universitários. Tese de Doutorado em Educação. Universidade Estadual de Campinas, Campinas.

Cerqueira, T. C. S. \& Santos, A. A. A. (2000). As possibilidades de avaliação dos estilos de aprendizagem em universitários. Em F. F. Sisto, E. T. B. Sabardelini \& R. Primi (Orgs.), Contextos e questões da avaliação psicológica (pp. 155-172). São Paulo: Casa do Psicólogo.

Corrêa, M. C. P. (2002). No ritmo do iê-iê-iê. AMAE Educando, $X X X V(306), 30-32$.

Honigsfeld, A. \& Dunn, R. (2003). High school male and female learning style similarities and differencesin diverse nations. Journal of Educational Research, 96(4), 195-206.

Figueiredo, E. L. (1996). Evolução do pensamento criador em situação musical. Tese de Doutorado em Educação. Universidade Estadual de Campinas, Campinas.

Jongsma, K. S. (1990). Learning styles. The Reading Teacher, 4, 696-697.

Marquetti, G. P. (1994). Tchaikovski no recreio da pré-escola: um estudo observacional do comportamento de crianças durante o recreio e em sala de aula. Dissertação de Mestrado em Psicologia. Universidade de São Paulo, São Paulo.
Messick, S. (1994). The matter of style: Manifestations of personality in cognition, learning, and teaching. Educational Psychologist, 29(3), 121-136.

Montanari, V. (1993). História da música: da idade da pedra à idade do rock ( $2^{\mathrm{a}}$ ed.). São Paulo:Ática.

Nezu, A. M. \& Nezu, C. M. (1999). Treinamento em solução de problemas. Em V. E. Caballo (Org.), Manual de técnicas de terapia e modificação do comportamento (pp. 471-493). São Paulo: Santos.

Pellegrini, D. (2000). O fã-club mirim de Fígaro. Nova Escola, 15(137), 2-3.

Pitta, K. B., Santos, L. A. D., Escher, C. A. \& Bariani, I. C. D. (2000). Estilos cognitivos de estudantes de psicologia: impacto da experiência em iniciação científica. Psicologia Escolar $e$ Educacional, 4(2), 41-49.

Riding, R. J. \& Cheema, I. (1991) Cognitive styles: an overview and integration. Educational Psychology, 11, 193-215.

Riding, R. J., Grimley, M., Dahraei, H. \& Banner, G. (2003). Cognitive style, working memory and learning behaviour and attainment in school subjects. British Journal of Educational Psychology, 73, 149-169.

Rosa, C. O. Z. \& Santos, A. A. A. (2001). Estilos de aprendizagem e escolha de carreira entre estudantes de medicina. Boletim de Psicologia, 51(114), 81-96.

Salvucci, M. (1997). O brincar de pensar dentro da sala de aula. Revista de Educação, 1(3), 75-76.

Santos, A. A. A., Bariani, I. C. D. \& Cerqueira, T. C. S. (2000). Estilos cognitivos e estilos de aprendizagem. Em F. F. Sisto, G. C. Oliveira \& E. L. T. Fini (Orgs.), Leituras de psicologia para a formação de professores (pp. 44-57). Petrópolis: Vozes.

Schmeck, R. R. \& Geisler-Brenstein, E. (1989). Individual differences that affect the way students approach learning. Learning and Individual Differences, 1(1), 85-124.

Silva, A. V. E. (1998). Cantar para aprender. Nova Escola, XIII (133), 46-47.

Squarizi, L. (1999). Escala sobre estilos de pensar e aprender. Dissertação de mestrado. Pontifícia Universidade Católica de Campinas, Campinas.

Enviado: $27 / 04 / 2005$ Revisado: $10 / 07 / 2005$ Aceito: $15 / 07 / 2005$

\section{Sobre as autoras:}

Acácia Aparecida Angeli dos Santos: Psicóloga. Doutora em educação pela Universidade de São Paulo e docente do curso de psicologia e do Programa de Pós-Graduação Stricto Sensu em Psicologia da Universidade São Francisco. Bolsista produtividade do CNPq.

Endereço para correspondência: Programa de Pós-Graduação Stricto Sensu em Psicologia - Universidade São Francisco - Campus Itatiba 13251-040 - Itatiba-SP.

Rogéria Gasparim Amadi: Psicóloga escolar graduada pela Universidade São Francisco.

Katya Luciane de Oliveira: Psicóloga. Mestre em psicologia pelo Programa de Pós-Graduação Stricto Sensu em Psicologia da Universidade São Francisco. Doutoranda em Psicologia, Desenvolvimento Humano e Educação pela Faculdade de Educação da Unicamp. Docente dos cursos de Psicologia da Universidade de Alfenas e da Universidade São Francisco. 